\title{
Avaliação do impacto da hiperglicemia hospitalar em relação à morbimortalidade em pacientes não críticos
}

\author{
Impact assessment of hospital hyperglycemia on morbidity and mortality in noncritical \\ patients
}
Evaluación del impacto de la hiperglucemia hospitalaria en la morbilidad y mortalidad en pacientes no críticos

Rudival Faial de Moraes Júnior ${ }^{1 *}$, Ricardo Chaves Branco ${ }^{2}$, William Rodrigues Costa ${ }^{2}$.

\begin{abstract}
RESUMO
Objetivo: Avaliar o impacto da hiperglicemia hospitalar nas taxas de mortalidade, tempo de internação e necessidade de suporte em unidade de terapia intensiva (UTI) nos pacientes não críticos hospitalizados nas enfermarias de clínica médica de um hospital público do Pará. Métodos: Estudo quantitativo, transversal, com amostra de 187 pacientes. Os grupos com e sem hiperglicemia foram avaliados quanto ao tempo de internação, necessidade de internação em UTI e óbito. Resultados: Não foram obtidas diferenças estatisticamente significativas que relacionassem a hiperglicemia hospitalar com o prolongamento no tempo de internação, maior necessidade de suporte em UTI e maior mortalidades dos pacientes. Notou-se, porém, que houve uma maior mortalidade entre os pacientes com diagnóstico de hiperglicemia hospitalar sem diagnóstico prévio de diabetes, mesmo que sem diferença estatisticamente significativa. Conclusão: Apesar da literatura relacionar a hiperglicemia com desfechos adversos, o presente estudo não mostrou diferença estatisticamente significativa em relação a duração do tempo de internação, necessidade de atendimento em UTI e mortalidade.
\end{abstract}

Palavras-chave: Hiperglicemia, Unidade terapia intensiva, Mortalidade.

\begin{abstract}
Objective: To evaluate the impact of hospital hyperglycemia on mortality rates, length of stay and need for intensive care unit (ICU) support in non-critically ill patients hospitalized in the medical clinic wards of a public hospital in Pará. Methods: Quantitative, cross-sectional study, with a sample of 187 patients. The groups with and without hyperglycemia were evaluated for length of stay, need for ICU stay and death. Results: No statistically significant differences were found that related hospital hyperglycemia with prolongation of hospitalization, greater need for ICU support and greater death of patients. However, it was noted that there was a higher mortality among patients diagnosed with hospital hyperglycemia without previous diagnosis of diabetes, even without statistically significant difference. Conclusion: Although the literature relates hyperglycemia with adverse outcomes, the present study did not show a statistically significant difference regarding length of stay, need for ICU care and mortality.
\end{abstract}

Keywords: Hyperglycemia, Intensive care unit, Mortality.

\section{RESUMEN}

Objetivo: Evaluar el impacto de la hiperglucemia hospitalaria en las tasas de mortalidad, la duración de la estadía y la necesidad de apoyo de la unidad de cuidados intensivos (UCl) en pacientes no críticos hospitalizados en las salas de la clínica médica de un hospital público en Pará. Métodos: Estudio cuantitativo, transversal, con una muestra de 187 pacientes. Los grupos con y sin hiperglucemia fueron evaluados por la

\footnotetext{
${ }^{1}$ Fundação Santa Casa de Misericórdia do Pará (FSCMP), Belém-PA. *E-mail: rfaial@terra.com.br

${ }^{2}$ Acadêmico da Universidade do Estado do Pará (UEPA), Belém-PA.
} 
duración de la estadía, la necesidad de estadía en la UCI y la muerte. Resultados: No se encontraron diferencias estadísticamente significativas que relacionen la hiperglucemia hospitalaria con la prolongación de la hospitalización, una mayor necesidad de apoyo en la UCl y una mayor muerte de los pacientes. Sin embargo, se observó que hubo una mayor mortalidad entre los pacientes diagnosticados con hiperglucemia hospitalaria sin diagnóstico previo de diabetes, incluso sin una diferencia estadísticamente significativa. Conclusión: Aunque la literatura relaciona la hiperglucemia con los resultados adversos, el presente estudio no mostró una diferencia estadísticamente significativa con respecto a la duración de la estadía, la necesidad de atención en la $\mathrm{UCl}$ y la mortalidad.

Palabras-clave: Hiperglucemia, Unidad de cuidados intensivos, Mortalidad.

\section{INTRODUÇÃO}

A hiperglicemia hospitalar $(\mathrm{HH})$ tem como definição qualquer valor de glicemia superior de $140 \mathrm{mg} / \mathrm{dL}$ no paciente hospitalizado, sendo ela ainda pouco valorizada e inadequadamente diagnosticada e tratada em diversos centros hospitalares do Brasil e do mundo (UMPIERREZ GE, et al., 2012; GOMES PM, et al., 2014). Diversas pesquisas internacionais realizadas nas últimas duas décadas, período em que o interesse pelo tema se intensificou, sobretudo após a publicação do trabalho de Van Den Berghe G, et al. (2001), estabeleceram uma grande associação entre hiperglicemia em pacientes hospitalizados, com ou sem diabetes mellitus, e incremento do risco de complicações e morte (UMPIERREZ GE, et al., 2012; ERIKSSON M, et al., 2012; BONAMICHI BDSF, et al., 2015; PENNING S, et al., 2015).

Apesar da maioria dos estudos randomizados e controlados, que investigaram o impacto do tratamento da hiperglicemia, ter sido realizado em pacientes criticamente doentes, há dados observacionais que apoiam a importância do tratamento da hiperglicemia entre os pacientes não críticos (pacientes que não necessitam de cuidados em unidade de terapia intensiva), admitidos em enfermarias de clínica médica e cirurgia (KOSIBOROD M, et al., 2009; UMPIERREZ GE, et al., 2012). Nestes, a hiperglicemia está associada com internações mais prolongadas, aumento da incidência de infecções, bem como, maior incapacidade funcional após alta hospitalar e maior taxa de morte (BAKER EH, et al., 2006; UMPIERREZ GE, et al., 2012; MACALLISTER DA, 2014).

A real e exata incidência de hiperglicemia em pacientes hospitalizados é de difícil determinação, sobretudo devido a variação de acordo com a população estudada, gravidade da doença e definição utilizada (DAVIDSON P, et al., 2015). A HH engloba tanto pacientes portadores de diabetes prévio ou diagnosticados na internação, quanto indivíduos não diabéticos que apresentam hiperglicemia na presença de doenças agudas, a chamada hiperglicemia de estresse, fenômeno este bastante frequente (GOMES PM, et al., 2014).

O controle glicêmico é ponto fundamental do cuidado ao paciente hospitalizado independentemente da etiologia da hiperglicemia (KIRK JK e OLDHAM EC, 2010). Entretanto, Bogun M e Inzucchi SE (2013) afirmam que, na clínica médica e nos serviços de cirurgia, a hiperglicemia é frequentemente negligenciada ou manejada de forma insipiente. Corroborando este entendimento, há diversos estudos demonstrando que o manejo da hiperglicemia no hospital é geralmente considerado secundário em comparação com a condição que levou o paciente à internação (CRAWFORD K, 2013; GODINHO C, et al., 2015; MOREIRA ED, et al., 2013).

Diante disso, o presente estudo objetiva a avaliação do impacto da hiperglicemia hospitalar nas taxas de mortalidade, tempo de internação e necessidade de suporte em unidade de terapia intensiva nos pacientes hospitalizados nas enfermarias de clínica médica de um hospital do estado do Pará.

\section{MÉTODOS}

Foi realizado um estudo transversal e quantitativo, com pacientes internados nas enfermarias de clínica médica de um hospital do estado do Pará. Foram incluídos para análise todos os pacientes admitidos primariamente nos 46 leitos de internação nas enfermarias de clínica médica de um hospital do estado do Pará, no período de 15 de abril de 2017 a 15 de outubro de 2017. 
Os participantes foram estudados segundo os preceitos da Declaração de Helsinque e do Código de Nuremberg, sendo respeitadas as Normas de Pesquisa Envolvendo Seres Humanos (Res. CNS/MS 466/12) e suas complementares do Conselho Nacional de Saúde/Ministério da Saúde. A coleta dos dados foi realizada após aprovação pelo Comitê de Ética em Pesquisa, sob o ํo 66938117.5.0000.5171, com autorização da direção, mediante assinatura do Termo de Consentimento Livre e Esclarecido (TCLE) pelos participantes e assinatura de Termo de Confidencialidade de Utilização de Dados (TCUD) pelos autores.

Os dados foram coletados dos prontuários dos pacientes e todos estes tiveram a glicemia capilar aferida no momento da admissão, sendo distribuídos, de acordo com o valor obtido, em dois grupos: pacientes com $\mathrm{HH}$ e pacientes sem $\mathrm{HH}$. Os participantes foram avaliados em relação a tempo de internação, necessidade de suporte de terapia intensiva e óbito.

Utilizou-se para o diagnóstico de HH, o valor definido na diretriz de 2012 da Endocrine Society: glicemia capilar superior a 140 miligramas/decilitro $(\mathrm{mg} / \mathrm{dL})$. Foram considerados previamente diabéticos aqueles indivíduos com diagnóstico definido anteriormente à admissão. Hiperglicemia de estresse definida nos indivíduos que apresentaram glicemia capilar acima de $140 \mathrm{mg} / \mathrm{dL}$ associada à hemoglobina glicada inferior a 6,5\% e pacientes com diabetes oculto como aqueles sem histórico prévio e com hemoglobina glicada maior ou igual a 6,5\% (UMPIERREZ GE, et al., 2012; SBD, 2017). A dosagem de hemoglobina glicada foi realizada pelo laboratório do hospital, o qual utiliza o método considerado padrão-ouro pela American Diabetes Association, a cromatografia líquida de alta performance (ADA, 2018). A glicemia capilar foi realizada no

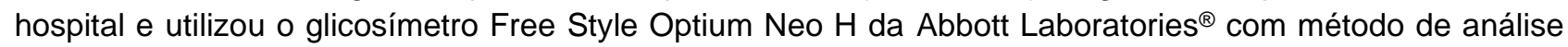
pela amperometria.

O tamanho amostral foi calculado através da fórmula para cálculo da amostra da distribuição normal, com base na população atendida nas enfermarias de clínica médica. Para a análise da significância estatística, foram aplicados os seguintes testes: Qui-Quadrado, teste $G$ de aderência para uma variável e independência na comparação entre duas variáveis categóricas e o teste t de Student para comparação entre as médias das variáveis numéricas. O nível de significância estatística adotado será alfa=0,05 ou $5 \%$, com intervalo de confiança de $95 \%$. Todas as informações coletadas foram armazenadas no software Excel $2007^{\circledR}$ (Microsoft Corporation, Redmond, USA) e analisadas por meio dos softwares BioEstat $5.3^{\circledR}$ e GraphPad Prism 5.0, incluindo a confecção dos gráficos.

\section{RESULTADOS}

Após análise dos critérios de inclusão e exclusão, foram incluídos 187 pacientes para a pesquisa. Destes, 111 eram do sexo masculino (59,4\%) e 76 do sexo feminino (40,6\%), com uma média de idade de 50,6 anos.

Dentre os 187 pacientes, 128 eram normoglicêmicos e 59 eram portadores de HH. Destes hiperglicêmicos, 39 eram portadores de diabetes diagnosticado previamente, 15 apresentavam hiperglicemia de estresse e 5 eram diabéticos sem diagnóstico prévio (diabetes oculto). A Tabela 1 ilustra algumas das principais variáveis avaliadas.

Tabela 1 - Principais resultados da pesquisa, clínica médica, hospital, abril-outubro, 2017.

\begin{tabular}{lc}
\hline Perfil & \\
\hline Total de pacientes & 187 \\
Idade & $50,6 \pm 19,5$ \\
Sexo (F/M) & $76(40,6 \%) / 111(59,4 \%)$ \\
IMC & $24,5 \pm 5,2$ \\
Hiperglicemia hospitalar & $59(31,6 \%)$ \\
Causas: Diabetes previamente & $39(66,1 \%)$ \\
Hiperglicemia de estresse & $15(25,4 \%)$ \\
Diabetes oculto & $5(8,5 \%)$ \\
Normoglicêmicos & $128(68,4 \%)$ \\
\hline
\end{tabular}

Nota: Dados são n (\%) ou média \pm desvio padrão (DP). Fonte: Moraes Júnior RF, et al., 2019. 
O tempo médio de internação hospitalar foi de 27,3 dias e não foi obtida diferença significante entre os grupos com e sem hiperglicemia $(p=0,1618)$.

Entre os participantes da pesquisa, 37 necessitaram de suporte em UTI e, dentre estes, 8 apresentaram $\mathrm{HH}$ e 29 eram normoglicêmicos. Porém, não houve associação significante entre internação na UTI e diagnóstico ou não de HH ( $p=0,1463)$ (Tabela 2).

Tabela 2 - Relação entre hiperglicemia hospitalar versus necessidade de internação em UTI, clínica médica, hospital, abril-outubro, 2017.

\begin{tabular}{lllll}
\hline \multirow{2}{*}{ Internação na UTI } & \multicolumn{5}{c}{ Hiperglicemia hospitalar } \\
\cline { 2 - 5 } & Sim & $\%$ & Não & $\%$ \\
\hline Sim & 8 & $21,6 \%$ & 29 & $78,4 \%$ \\
Não & 51 & $34,0 \%$ & 99 & $66,0 \%$ \\
\hline Total & $\mathbf{5 9}$ & $\mathbf{3 1 , 6 \%}$ & $\mathbf{1 2 8}$ & $\mathbf{6 8 , 4 \%}$ \\
\hline
\end{tabular}

$p=0,1463$ Teste Qui-Quadrado Independência.

Fonte: Moraes Júnior RF, et al., 2019.

Dos 41 pacientes que foram a óbito, 29 se apresentaram normoglicêmicos e 12 apresentaram HH. Entretanto, a associação entre HH e óbito não teve significância estatística $(p=0,8683)$ (Tabela 3).

Tabela 3 - Pacientes que evoluíram ou não a óbito com e/ou sem hiperglicemia hospitalar, clínica médica, hospital, abril-outubro, 2017.

\begin{tabular}{lllll}
\hline \multirow{2}{*}{ Evolução para óbito } & \multicolumn{4}{c}{ Hiperglicemia hospitalar } \\
\cline { 2 - 5 } & Sim & $\%$ & Não & $\%$ \\
\hline Sim & 12 & $29,3 \%$ & 29 & $70,7 \%$ \\
Não & 47 & $32,2 \%$ & 99 & $67,8 \%$ \\
\hline Total & $\mathbf{5 9}$ & $\mathbf{3 1 , 6 \%}$ & $\mathbf{1 2 8}$ & $\mathbf{6 8 , 4} \%$ \\
\hline
\end{tabular}

$p=0,8683$ Teste Qui-Quadrado Independência.

Fonte: Moraes Júnior RF, et al., 2019.

Dentre os pacientes que evoluíram a óbito, nota-se uma proporção maior entre os que não tinham diagnóstico prévio de diabetes, mas que tiveram $\mathrm{HH}(87,8 \%)$, sobre os pacientes que tinham diagnóstico prévio de diabetes $(12,2 \%)$. Estatisticamente sem associação significante $(p=0,1844)$ (Tabela 4).

Tabela 4 - Diagnóstico prévio de diabetes associados ou não à hiperglicemia hospitalar e sua relação com óbito, clínica médica, hospital, abril-outubro, 2017.

\begin{tabular}{lcccc}
\hline \multirow{2}{*}{ Hiperglicemia hospitalar + Diabetes } & \multicolumn{4}{c}{ Evolução para óbito } \\
\cline { 2 - 5 } & Sim & $\%$ & Não & $\%$ \\
\hline Sim (HH + DM) & 5 & $12,2 \%$ & 34 & $23,3 \%$ \\
Não (HH - DM) & 36 & $87,8 \%$ & 112 & $76,7 \%$ \\
\hline Total & $\mathbf{4 1}$ & $\mathbf{2 1 , 9 \%}$ & $\mathbf{1 4 6}$ & $\mathbf{7 8 , 1 \%}$ \\
\hline
\end{tabular}

$\mathrm{p}=0,1844$ Teste Qui-Quadrado Independência

Fonte: Moraes Júnior RF, et al., 2019. 
Dentre os atendidos na UTI que posteriormente evoluíram a óbito, 20\% tiveram diagnóstico de $\mathrm{HH}$, enquanto $80 \%$ eram normoglicêmicos. Entretanto, não se obteve associação independente significativa $(p=$ 0,2696) (Tabela 5).

Tabela 5 - Necessidade de UTI e evolução a óbito ou alta hospitalar versus hiperglicemia hospitalar, clínica médica, hospital, abril-outubro, 2017.

\begin{tabular}{lcccc}
\hline \multirow{2}{*}{ UTI + Evolução para óbito } & \multicolumn{4}{c}{ Hiperglicemia hospitalar } \\
\cline { 2 - 5 } Sim & Sim & $\%$ & Não & $\%$ \\
Não & 5 & $20,0 \%$ & 20 & $80,0 \%$ \\
\hline Total & 54 & $33,3 \%$ & 108 & $66,7 \%$ \\
\hline
\end{tabular}

$p=0,2696$ Teste Qui-Quadrado Independência

Fonte: Moraes Júnior RF, et al., 2019.

\section{DISCUSSÃO}

A hiperglicemia hospitalar ainda é uma condição extremamente negligenciada e em poucos hospitais brasileiros a triagem ativa desta condição é realizada. Segundo os dados do presente estudo, cerca $32 \%$ dos pacientes tiveram diagnóstico de HH. Destes, aproximadamente dois terços relatavam diabetes prévio, e um terço, não. Dos pacientes com HH sem histórico prévio de diabetes, quase $26 \%$ eram portadores de hiperglicemia de estresse e $8,5 \%$ de diabetes não diagnosticado. Umpierrez GE, et al. (2002) expõem um achado de 32 a $38 \%$ dos pacientes hospitalizados em enfermarias apresentam $\mathrm{HH}$ e, aproximadamente, um terço destes pacientes não apresentavam histórico anterior de diabetes.

Segundo vários estudos, os portadores de diabetes são mais propensos a serem hospitalizados, costumam ter internações mais prolongadas, assim como, necessitam de maior suporte clínico em unidade de terapia intensiva. A associação entre a ocorrência de hiperglicemia em pacientes hospitalizados, portadores ou não de diabetes, e aumento do risco de complicações e mortalidade está bem estabelecida (GALINDO R e UMPIERREZ GE, 2018; CHANG MW, et al., 2018).

O presente estudo não evidenciou diferença estatisticamente significativa em relação à duração do tempo de internação entre pacientes com e sem hiperglicemia $(p=0,1618)$, indicando assim que, provavelmente, a hiperglicemia per se não é fator independente de prolongamento de tempo de internação hospitalar. Outros fatores, como a variação glicêmica diária, podem estar implicados nessa condição, como mostrado por Mendez CE, et al. (2013), Akirov A, et al. (2017) e Sáenz-Abad D, et al. (2015). Tal variação, infelizmente, não foi avaliada neste estudo.

Também não se observou diferença entre os grupos quanto à necessidade de internação em UTI. Diferentemente dos achados de vários trabalhos como o de Van Vught LA, et al. (2017) e Krinsley JS, et al. (2017), que estabelecem firmemente associação entre $\mathrm{HH}$ e desfechos negativos, não foi encontrada uma associação estatisticamente significativa entre hiperglicemia hospitalar e mortalidade, seja em pacientes hospitalizados nas enfermarias ou nos que foram transferidos para a UTI. Entre os pacientes que foram atendidos em UTI e evoluíram a óbito, obteve-se um total de $20 \%$ com diagnóstico de $\mathrm{HH}$, enquanto $80 \%$ eram normoglicêmicos, com ausência de associação independente significativa entre tais fatores $(p=0,2696)$, contudo, cerca de $30 \%$ dos pacientes hospitalizados nas enfermarias que foram a óbito apresentavam hiperglicemia $(p=0,8683)$.

Os dados do atual estudo demonstraram que os pacientes com diagnóstico prévio de diabetes tiveram menor mortalidade, pois dos pacientes que evoluíram a óbito e que tinham hiperglicemia, mais de $87 \%$ apresentaram $\mathrm{HH}$ sem diagnóstico de diabetes previamente, contra cerca de $12 \%$ dos que evoluíram a óbito 
e tinham diabetes diagnosticado anteriormente. Segundo Umpierrez GE e Pasquel FJ (2017), a presença de diabetes previamente reduz a evolução com desfechos negativos para o paciente. Por outro lado, é bem estabelecido em várias publicações, que os portadores de hiperglicemia de estresse têm apresentado um risco maior de piores desfechos clínicos, em comparação a pacientes com diabetes diagnosticado previamente (GALINDO RJ e UMPIERREZ GE, 2018; DESILLES J, et al., 2013).

Corroborando tais achados, Carrasco-Sánchez FJ, et al. (2018) demonstraram que a incidência de complicações foi diretamente associada à gravidade da hiperglicemia em pacientes sem diabetes, especificamente a hiperglicemia induzida pelo estresse, sendo que $14 \%$ dos pacientes com hiperglicemia necessitaram de atendimento na UTI, concordando assim com o presente trabalho, o qual identificou que, dos pacientes que necessitaram de internação na UTI, 21,6\% apresentavam HH sem diabetes previamente, porém esta associação não foi estatisticamente significativa $(p=0,1463)$.

Pieralli $F$, et al. (2015) evidenciam que dentre os pacientes não críticos que foram a óbito, a percentagem foi maior no grupo entre os pacientes com $\mathrm{HH}$ sem diagnóstico prévio de diabetes (7,9\%), quando comparado aos que foram a óbito e tinham diagnóstico de diabetes $(5,7 \%)$, novamente corroborando o provável fator protetor que o diabetes estabelecido tem nos pacientes internados, sobretudo nos que evoluem para a UTI.

Talvez o número de pacientes avaliados tenha sido um fator que contribuiu para não observarmos uma associação significativa entre hiperglicemia e desfechos adversos, assim como, o protocolo para o diagnóstico e tratamento da hiperglicemia hospitalar que fora introduzido no hospital do estudo há quase uma década.

\section{CONCLUSÃO}

A HH é uma condição extremamente comum, porém inadequadamente diagnosticada e tratada na maioria dos centros hospitalares, e diversos estudos têm demonstrado a associação direta desta com maior tempo de hospitalização, necessidade de suporte em UTI e taxa de óbito. Apesar de a literatura relacionar a hiperglicemia a tais desfechos, este estudo não mostrou tal correlação. Contudo, notou-se que a hiperglicemia hospitalar sem diagnóstico prévio da diabetes foi associada a uma tendência maior de mortalidade intrahospitalar. Muito provavelmente, a introdução de protocolo para o diagnóstico e tratamento da HH implantado no hospital foi o principal fator responsável pelos resultados encontrados.

\section{AGRADECIMENTOS}

Agradecemos ao hospital, que permitiu tal pesquisa em seu interior, e à estatística Mariseth Carvalho de Andrade, que nos auxiliou enormemente na construção deste trabalho.

\section{REFERÊNCIAS}

1. AKIROV A, et al. High glucose variability increases mortality risk in hospitalized patients. The Journal of Clinical Endocrinology and Metabolism, 2017; 102 (7): 2230-2241.

2. AMERICAN DIABETES ASSOCIATION. Diabetes care in the hospital: standards of medical care in diabetes -2018 . Diabetes Care, 2018; 41 (1): 144-151.

3. BAKER EH, et al. Hyperglycaemia is associated with poor outcomes in patients admitted to hospital with acute exacerbations of chronic obstructive pulmonary disease. Thorax, 2006; 61: 284-289.

4. BOGUN M, INZUCCHI SE. Inpatient management of diabetes and hyperglycemia. Clinical Therapeutics, $2013 ; 35$ (5): 724-733.

5. BONAMICHI BDSF, et al. Aplicabilidade clínica da hemoglobina glicada na evolução do paciente com hiperglicemia hospitalar. Revista da Sociedade Brasileira de Clínica Médica, 2015; 13 (2): 111-113.

6. CARRASCO-SÁNCHEZ FJ, et al. Hiperglucemia inducida por estrés en pacientes ancianos hospitalizados no críticos. Revista Clínica Española, 2018; 218 (5): 223-231.

7. CHANG MW, et al. Stress-induced and diabetic hiperglycemia associated with higher mortality among intensive care unit trauma patients: cross-sectional analysis of the propensity score-matched population. International Journal of Environmental Research and Public Health, 2018; 15: 992.

8. CRAWFORD K. Guidelines for care of the hospitalized patient with hyperglycemia and diabetes. Critical Care Nursing Clinics of North America, 2013; 25 (1): 1-6. 
9. DAVIDSON P, et al. Management of hyperglycemia and enteral nutrition in the hospitalized patient. Nutrition in Clinical Pratice, 2015; 30 (5): 652-659.

10. DESILLES JP, et al. Diabetes mellitus, admission glucose, and outcomes after stroke thrombolysis: a registry and systematic review. Stroke, 2013; 44 (7): 1915-1923.

11. ERIKSSON M, et al. The disparity in long-term survival after a first stroke in patients with and without diabetes persists: the Northern Sweden MONICA study. Cerebrovascular Diseases, 2012; 34 (2): 153-160.

12. GALINDO RJ, UMPIERREZ GE. Endocrine and metabolic medical emergencies: a clinician's guide. Management of diabetes and/or hyperglycemia in non-critical care hospital settings. 2nd ed. New Jersey; Willey, 2018; 491-505 p.

13. GODINHO C, et al. Joint recommendations of the Portuguese Diabetology Society (SPD) / Portuguese Internal Medicine Society (SPMI) on the management and treatment of hyperglycemia in non-critical hospital inpatients. Revista Portuguesa de Diabetes, 2015; 1: 18.

14. GOMES PM, et al. Controle da hiperglicemia intra-hospitalar em pacientes críticos e não-críticos. Medicina (Ribeirão Preto.Online), 2014; 47 (2): 194-200.

15. KIRK JK, OLDHAM EC. Hyperglycemia management using insulin in the acute care setting: therapies and strategies for care in the non-critically ill patient. Annals of Pharmacotherapy, 2010; 44: 1222-1230.

16. KOSIBOROD M, et al. Elevated admission glucose and mortality in elderly patients hospitalized with heart failure. Circulation, 2009; 119: 1899-1907.

17. KRINSLEY JS, et al. Glucose control, diabetes status, and mortality in critically ill patients: the continuum from intensive care unit admission to hospital discharge. Mayo Clinic Proceedings, 2017; 92 (7): 1019-1029.

18. MACALLISTER DA. Stress hyperglycaemia in hospitalised patients and their 3-year risk of diabetes: a scottish reptrospective cohort study. PLOS Medicine, 2014; 11 (8): 1-18.

19. MENDEZ CE, et al. Increased glycemic variability is independently associated with length of stay and mortality in noncritically ill hospitalized patients. Diabetes Care, 2013; 36: 4091-4097.

20. MOREIRA ED, et al. Glycemic control and diabetes management in hospitalized patients in Brazil. Diabetology \& Metabolic Syndrome, 2013; 5: 02-08.

21. PENNING S, et al. Glucose control positively influences patient outcome: a retrospective study. Journal of Critical Care, 2015; 30 (3): 455-459.

22. PIERALLI F, et al. The classification of hospitalized patients with hyperglycemia and its implication on outcome: results from a prospective observational study in Internal Medicine. Internal and Emergency Medicine, 2015; 11 (5): $649-656$.

23. SOCIEDADE BRASILEIRA DE DIABETES. Diretrizes da Sociedade Brasileira de Diabetes 2017-2018. SBD, 2017: 353-357.

24. SÁENZ-ABAD D, et al. Importancia pronóstica de la variabilidade glucémica sobre la mortalidad intrahospitalaria. Revista Clínica Española, 2015; 215 (9): 479-485.

25. UMPIERREZ GE, PASQUEL FJ. Management of inpatient hyperglycemia and diabetes in older adults. Diabetes Care, 2017; 40 (4): 509-517.

26. UMPIERREZ GE, et al. Management of hyperglycemia in hospitalized patients in non-critical care setting: an endocrine society clinical practice guideline. The Journal of Clinical Endocrinology and Metabolism, $2012 ; 97$ (1): 1638.

27. UMPIERREZ GE, et al. Hyperglycemia: an independent marker of in-hospital mortality in patients with undiagnosed diabetes. The Journal of Clinical Endocrinology and Metabolism, 2002; 87: 978-982.

28. VAN DEN BERGHE G, et al. Intensive insulin therapy in critically ill patients. The New England Journal of Medicine, $2001 ; 345$ (19): 1359-1367.

29. VAN VUGHT LA, et al. Diabetes is not associated with increased 90-day mortality risk in critically ill patients with sepsis. Critical Care Medicine, 2017; 45 (10): 1026-1035. 